\title{
Investigando el entrepreneurship tras un marco teórico y su aporte al desarrollo económico de Colombia
}

John Orlando Crissien Castillo*

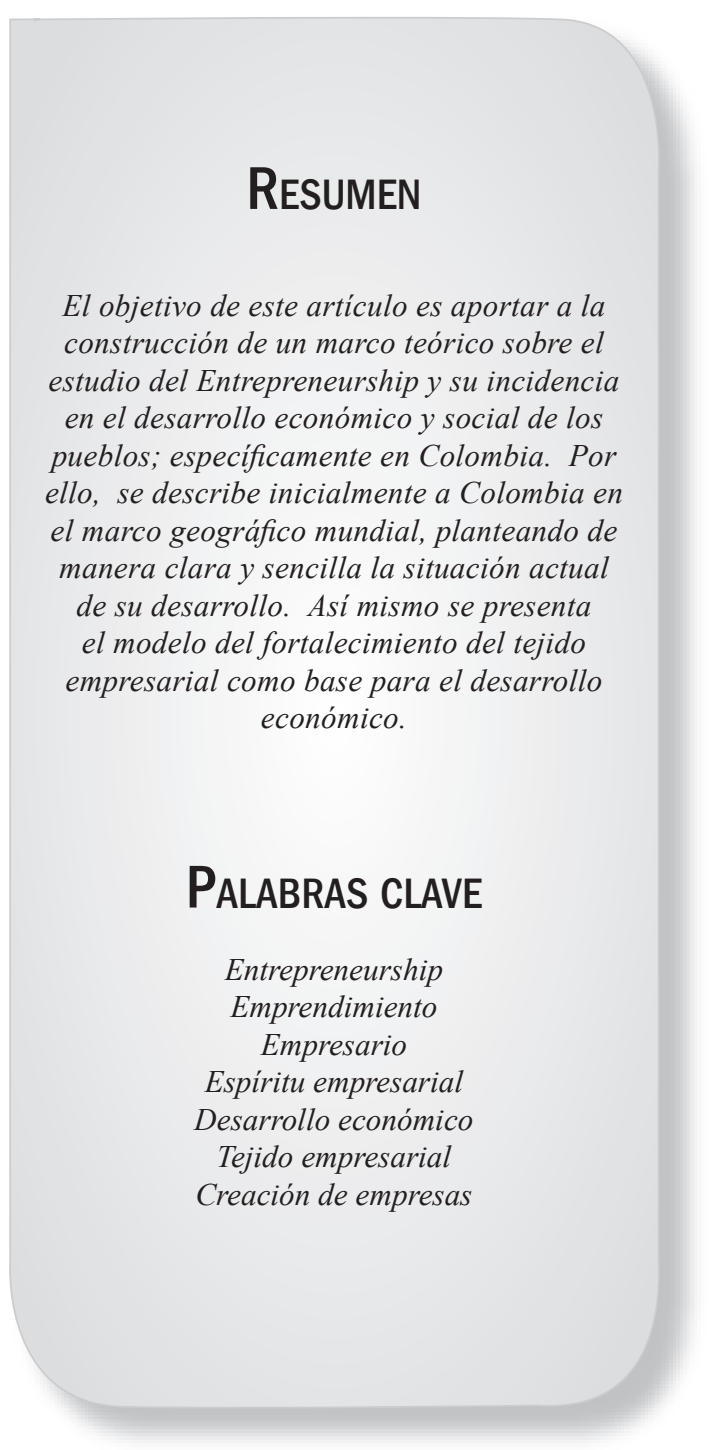

\section{Introducción}

$\mathrm{P}$

resentando el entrepreneurship, el emprendimiento o al empresario como estrategia de desarrollo económico; el artículo se concentra en definir dicho fenómeno desde la óptica del marco teórico. Inicialmente presentando un acercamiento a las definiciones desde el punto de vista de las palabras más utilizadas, los temas investigados y los roles asignados por los investigadores del fenómeno.

Posteriormente se presentan dos enfoques investigativos relevantes con un acercamiento a Veciana y a Kantis. Finalmente, se describen las definiciones y orientaciones de teóricos relevantes en el estudio del fenómeno del entrepreneurship.

* Doctorando en Ciencias Empresariales de la Universidad Antonio de Nebrija España, MBA,Master of Business Administration, WIU. EEUU, MAD Master en Alta Direccion, Colegio de Graduados en Alta Direcion, Mexico D.F. Administrador de Empresas EAN, Colombia. Profesor Asociado Facultad de PostgradosConsejero, Universidad EAN, Colombia. 


\section{Entrepreneurship y desarrollo económico en Colombia \\ Colombia}

\subsection{Situación actual}

Desarrollo económico y social de los pueblos. Esta frase es el detonante de este proyecto de investigación. Colombia es un país en vías de desarrollo, una economía emergente, localizada geográficamente en la parte norte de Latinoamérica.

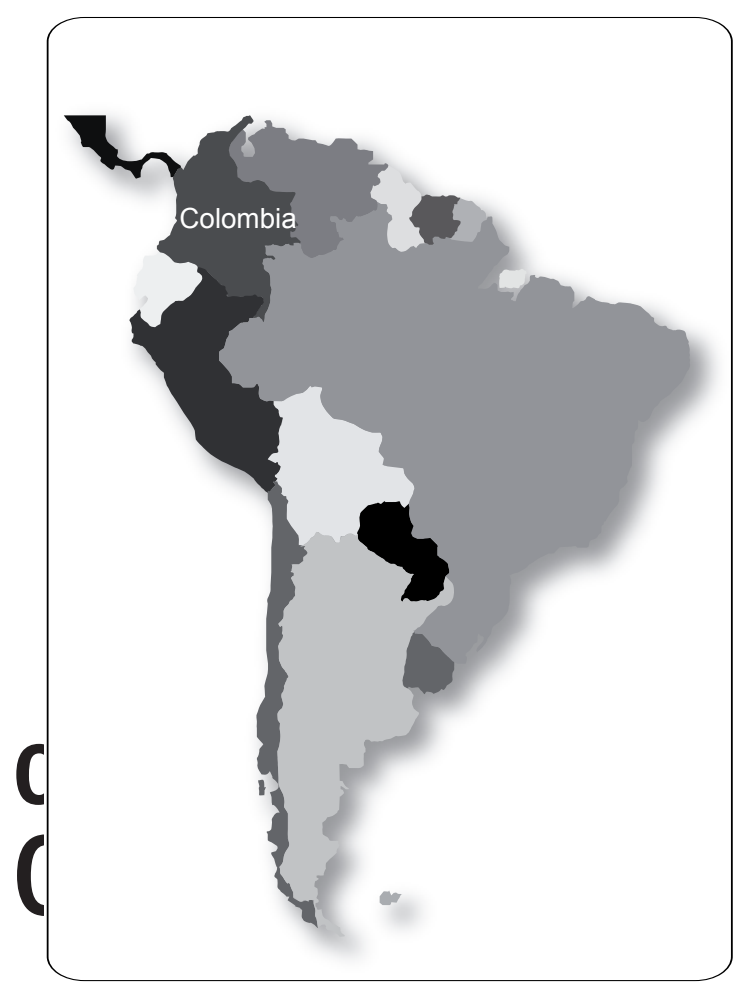

Fuente. http://www.google.com.co

\section{Abstract}

This article aims at making a theoretical framework to study entrepreneurship and its incidence in the social and economic development of countries, specifically Colombia. In it, the author describes Colombia from a geographical point of view and clearly states its current developmental situation. Besides, an entrepreneurial model for the strengthening of organizational network for economic development is also presented here.

\section{Key Words}

Entrepreneurship Manager

Entrepreneurial spirit Economic development Organizational network

Company creation 
Aun cuando el desarrollo económico, medido por su crecimiento, ha sido evidente en los últimos años, en términos de calidad de vida de sus ciudadanos debe mejorar en los próximos años. El objetivo final del desarrollo económico, es el mejoramiento de la calidad de vida de los habitantes; aun cuando, subjetivo, la calidad de vida puede y debe medirse en aspectos tales como: el acceso y cobertura en salud, educación, fuentes de trabajo, desarrollo de la libre personalidad, posibilidad de tener una vivienda digna y en general, el desarrollo humano y su bienestar.

El cómo lograr este desarrollo y crecimiento económico, orienta el estudio a las diferentes teorías económicas. La teoría económica institucional, específicamente en la vertiente liderada por North (1990) es la escogida como marco general de estudio. El Gobierno colombiano desde mediados de los años noventa, estableció leyes que propenden por el desarrollo y creación de empresas. A partir de dichas leyes se han creado organismos y programas orientados al apoyo de potenciales empresarios. En la actualidad existen normas generales y específicas así como leyes y medidas de apoyo a la creación de empresas que por estar tan dispersas son poco conocidas. (Gómez Liyis 2006).

Colombia es un país de contrastes, con abundantes recursos naturales pero con poca capacidad para producir riqueza. Su organización social es limitada para restringir las acciones de los individuos. Sus arreglos institucionales impiden la suficiente acumulación de capital y fallan al proveer la seguridad legal, los incentivos económicos y las formas de participación social necesarias para un desarrollo económico sostenible (Kalmanovitz, 2001: 89). La creación de nuevas empresas, como elemento clave del desarrollo económico, también es producto de las características institucionales prevalecientes, de sus limitaciones formales e informales y de sus mecanismos de regulación. Dicho de otra manera, el nacimiento de nuevas empresas requiere determinadas condiciones políticas, sociales y económicas para que ello sea posible. (Gómez Liyis 2006).

La situación problémica es la siguiente. El desarrollo económico se logra por la vía de la creación de empresas. Lo que se ha denominadoel tejido empresarial. Nuevos empresarios crean nuevas empresas. Empresarios con capacidad gerencial transforman empresas pequeñas, en empresas medianas y grandes, las cuales consolidadas competitivamente a nivel nacional e internacional, generan bienestar a los grupos de interés stake holders, incluyendo al Estado que recibe ingresos por la vía de los impuestos. De esta manera se genera un bosque de empresas que aportan de manera sistemática y constante al aparato económico de un país generando bienestar y desarrollo económico. 
Gráfica 1. Desarrrollo económico y educaciónempresarial

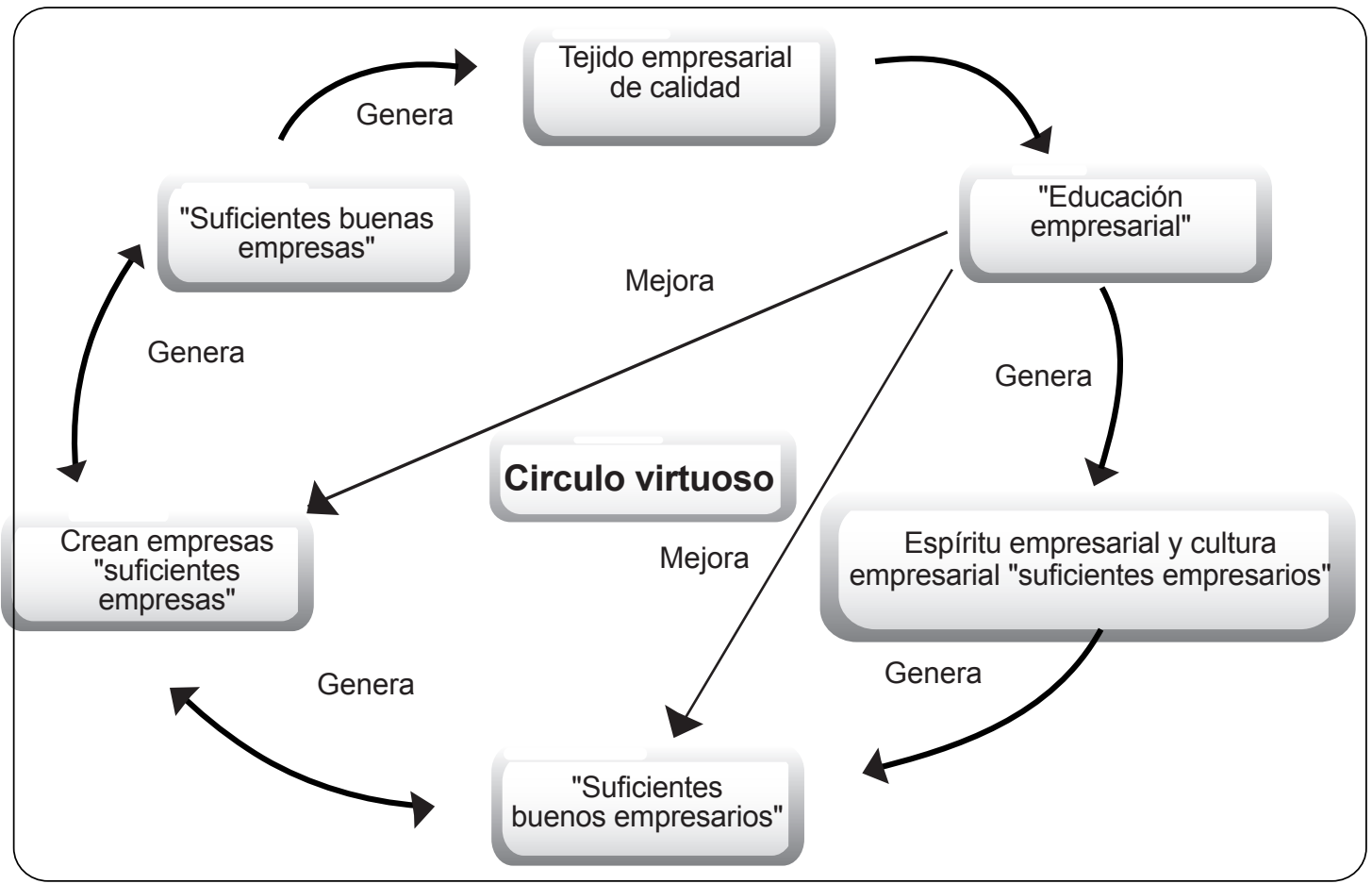

Para Schumpeter el cuarto factor de la economía, se denomina el empresario. El innovador destructivo, quien genera cambios en los mercados, en las tecnologías, en definitiva, quien crea una empresa.

Partiendo de esta premisa, varias preguntas surgen.

$\gg$ ¿Quién es un empresario?

\section{codecer Colombia}

$>$ ¿Nace o se hace?
$>$ ¿Cuál es la definición de nuestro individuo de estudio?

$>$ ¿Empresario?

$>$ ¿Emprendedor?

$>$ ¿Intraempresario?

$\supset$ ¿Líder?

$>$ ¿Cuál es el campo de estudio:emprendimiento, espírituempresarial creación de empresas, emprendedorismo, empresarismo, empresariado, entrepreneurship, cultura empresarial, administración de empresas? 


\section{Aspectos conceptuales}

\subsection{Definiciones relevantes}

...Researching entrepreneurship is fun, fascinating, frustraiting-andimportant..... One of the fascinations is the richness of the phenomenon, which leads to one of the greatests frustrations, namely the lack of common understanding of what precisely entrepreneurship is.... (Davidsson Per, p. 1., 2005).

La investigación en entrepreneurship es divertida, fascinante, frustrante y al mismo tiempo importante. Una de las fascinaciones más importantes es la riqueza del fenómeno, lo cual genera una de las más grandes frustraciones, también nombrada como la falta de entendimiento del fenómeno mismo.

\section{$>$ ¿Qué es?}

Iniciando este estudio de investigación, es importante entonces generar un marco conceptual sobre entrepreneurship como objeto de estudio. La primera consideración es la misma palabra entrepreneurship. Esta palabra no existe en el diccionario de la Real Academia de la Lengua Española ${ }^{1}$. ¿Entonces, cuál es el tema de investigación? Con este objetivo en mente se generó por parte del autor un mapa mental ${ }^{2}$ en el cual se concentran, autores relevantes y sus aportes no sólo a la definición de este tema, sino a aspectos relevantes que se incluyen en el fenómeno.

La construcción se generó basado en los autores citados por (Davidsson 2005 p, 1 a 7), y en investigación científica de los principales journals especializados.

> Nueva entrada (Lumpkin \& Dess, 1996)

> La creación de una nueva empresa (Low \& Mc Millan, 1988).

> Lacreación de nuevas organizaciones (Gartner, 1988).

DUna actividad con propósito de iniciar, mantener o agrandar un negocio 0 empresa orientada al lucro (Cole, 1949).

D Aprovechar la ventaja de una oportunidad, mediante la combinación de recursos, con los cuales se impacta un mercado (Wiklund, 1998).

\footnotetext{
La palabra entrepreneurship no está en el diccionario. http://buscon.rae.es/draeI/SrvltConsulta?TIPO_ BUS $=3 \& L E M A=$ entreprneurship

Mapa mental: es una representacion nemotécnica que simula las redes neuronales y el pensamiento irradiante. Los mapas mentales fueron desarrollados como técnica por Tony Buzan y el programa en el cual se generan se denomina Mind Manager X5.
} 
$>$ El proceso mediante el cual, individuos; ya sea por iniciativa propia o dentro de una organización aprovechan oportunidades sin importar los recursos sobre los cuales en el momento tienen control. (Stevenson \& Jarillo, 1990).

$>$ El proceso de crear algo nuevo o diferentequegenera valor invirtiendo todo el tiempo y esfuerzo necesario; asumiendo el riesgo financiero, psicológico y social y recibiendo los beneficios resultantes en términos monetarios y personales del mismo. (Hisrisch \& Peters, 1989).

En el mapa mental se presentan los acercamientos de Kizner (1983) y su orientación referente a los roles o acciones que los teóricos económicos le han asignado al fenómeno. De otra parte Gartner (1990), tiene un acercamiento hacia el fenómeno de investigación, enfocado a los temas centrales que se tocan en investigación.

Mapa mental 1

¿Que es entrepreneurship?

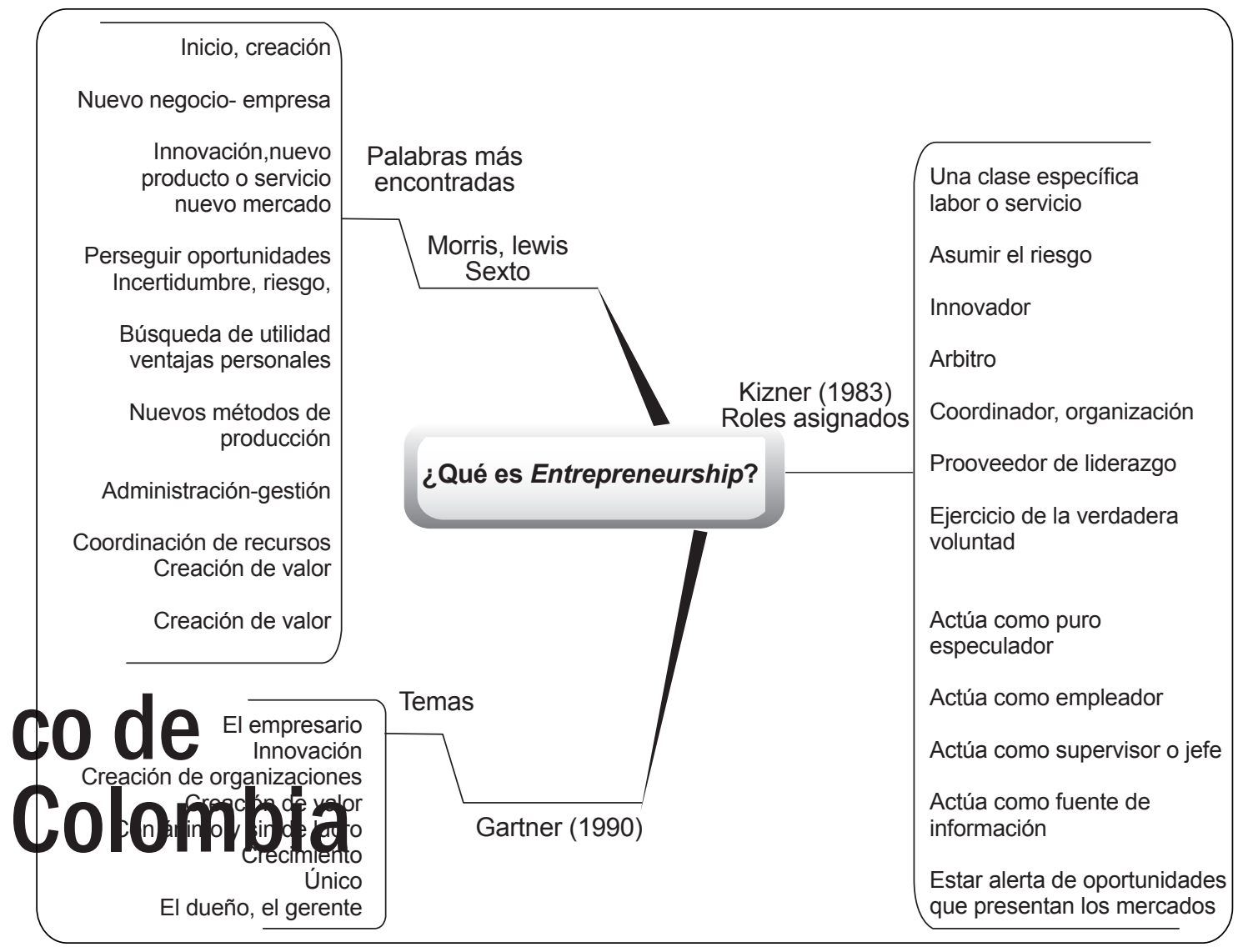

Fuente. Elaboración propia 
De manera similar el análisis de diferentes journals y libros realizado por Morris, Lewis \& Sexton (1994) generaron las palabras más comunes utilizadas para su definición.

En español entonces, en el marco de este estudio entrepreneurship se conceptualiza así:

Un individuo que emprende, crea una empresa y se torna empresario. El proceso general se denomina creación de empresas y la acción se denomina emprendimiento.

\subsection{Enfoques investigativos en entrepreneurship}

Dos referencias importantes que se deben tener en cuenta para generar un marco referencial teórico y conceptual desde los diferentes enfoques para la inves-tigación en creación de empresas son: Veciana ( 1999) y Kantis (2001), entre otros. La creación de empresas como objeto de estudio científico o como programa de investigación científica, tiene aproximadamente 50 años. Este es el periodo de desarrollo, en el cual básicamente el empresario se ha tornado como individuo a estudiar la creación y puesta en marcha de la empresa o start up y su consolidación.
Los factores y variables que afectan de manera positiva o negativa estos actores son fuente de investigación. Es así como desde ese centro de atención se han generado ciertos matices en investigación denominamos enfoques.

\subsubsection{Veciana y su perspectiva}

El mapa mental 2, presenta un diseño propio del autor como resumen de los aspectos analizados partiendo de la propuesta de Veciana en la Revista Europea de Dirección y Economía de la Empresa.

Los cuatro enfoques planteados acercan a posibilidades de investigación más puntuales, dependiendo de las hipótesis, de los gustos y preferencias de los investigadores.

En nuestro caso, el querer profundizar en los detonadores para la creación de empresas, modelos de rol, formación universitaria empresarial y desarrollo económico; tocan tanto el enfoque económico, como el enfoque sociocultural o institucional.

El enfoque sociocultural o institucional afecta directamente a los individuos, que más allá de perfiles y características investigadas en el enfoque psicológico es una persona con potencial infinito. 


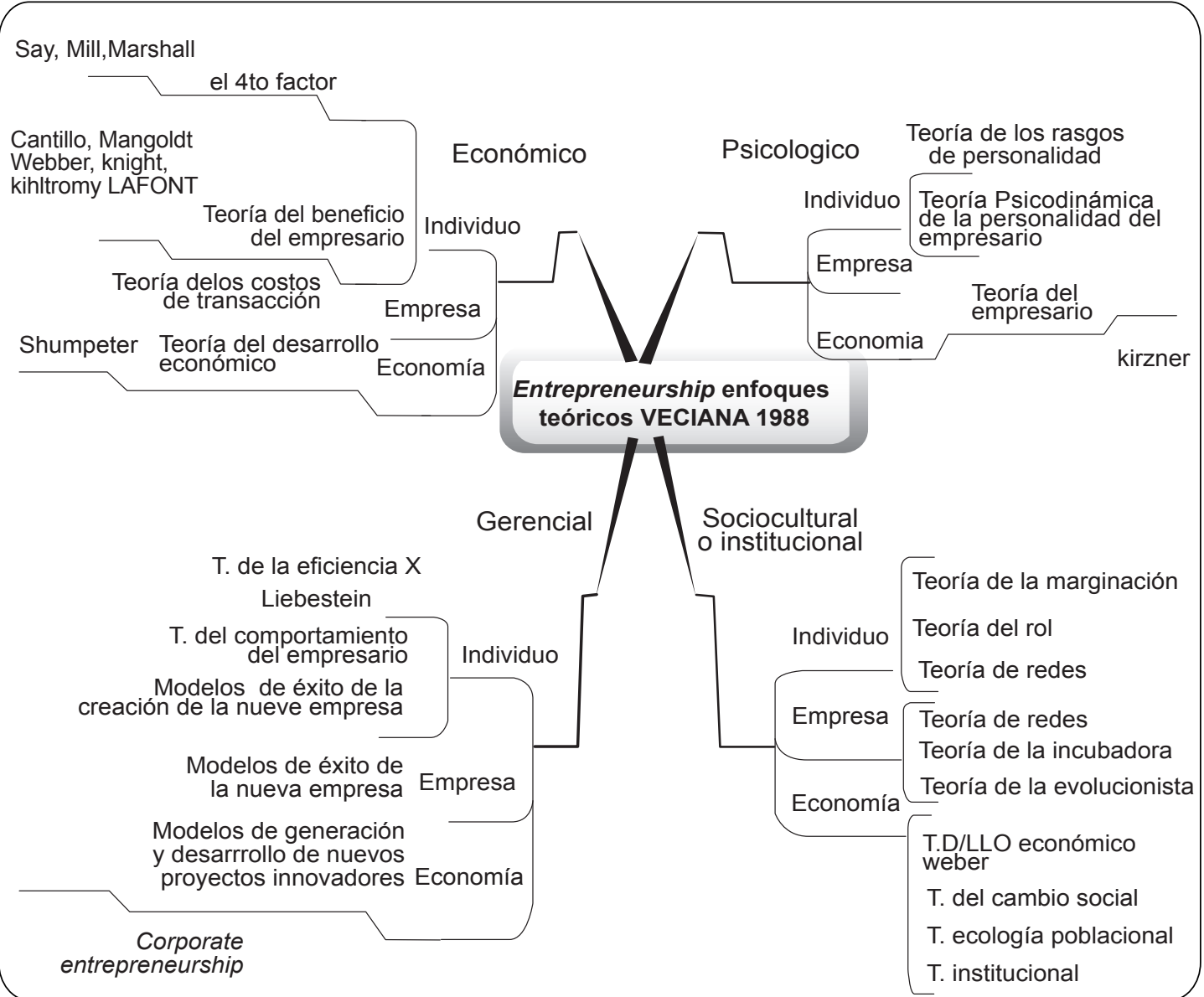

Elaboración propia. Fuente veneciana

Su entorno lo moldea, su enfoque ante la vida le da dirección; por esta causa la teoría del rol, teoría de la marginación y teoría de redes, desde el punto de vista del Individuo enmarcan teóricamente este estudio.

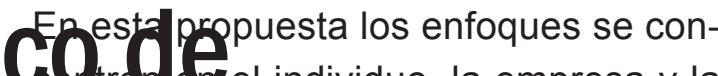

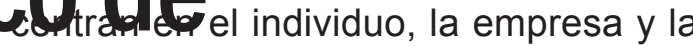

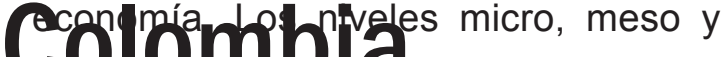
económica y gerencial más que una orientación psicológica, como la de McLelland (1961).

\subsubsection{Kantis y su perspectiva}

Si bien Veciana genera cuatro enfoques investigativos. Kantis (2001) a su vez, con sus compañeros Angelelli y Moori plantea que la creación de empresas y el marco teórico de estudio del empresario se enmarca en tres enfoques.

\section{> El enfoque económico odecondiciones} positivas o negativas, que coadyuvan a la creación de empresas de manera externa, que incluyen los factores de funcionamiento de los mercados, las condiciones económicas internas 
y externas de los estados, de los gobiernos que generan posibilidades motivantes para que el empresario emprenda y cree nuevas empresas. Si hay un mercado y se tienen los recursos para diseñar productos y servicios es natural que alguien tome esa oportunidad.

$>$ El enfoque no económico, en donde las características de personalidad hacen que el empresario surja aún en economías y mercados no positivos para el empresariado. La cultura o los factores socioculturales que proponen el ser empresario como legitima profesión; la legitimizacion del empresario afecta de manera directa la opción de crear empresa como proyecto de vida. La movilidad social por la vía de la independencia económica del empresario es otro de los enfoques teóricos que plantea Kantis.
DEl proceso empresarial, la empresa como fin no como medio del empresario para cumplir su proyecto de vida es el centro de este enfoque. El contexto y el proceso. En este se concentran teorías claras y específicas como el conductismo de Gardner (1990), Shapero y el proceso empresarial, GIBB (2004) con el entorno favorable desde la universidad.

En definitiva los enfoques planteados por Kantis Generan una claridad en términos de marco teórico para el estudio de este fenómeno. El enfoque no económico, donde la legitimización del empresario, la movilidad social y la marginalidad e integración social son los focos en los cuales se enmarcan los detonadores planteados: primero los modelos de rol y segundo la universidad con formación empresarial:

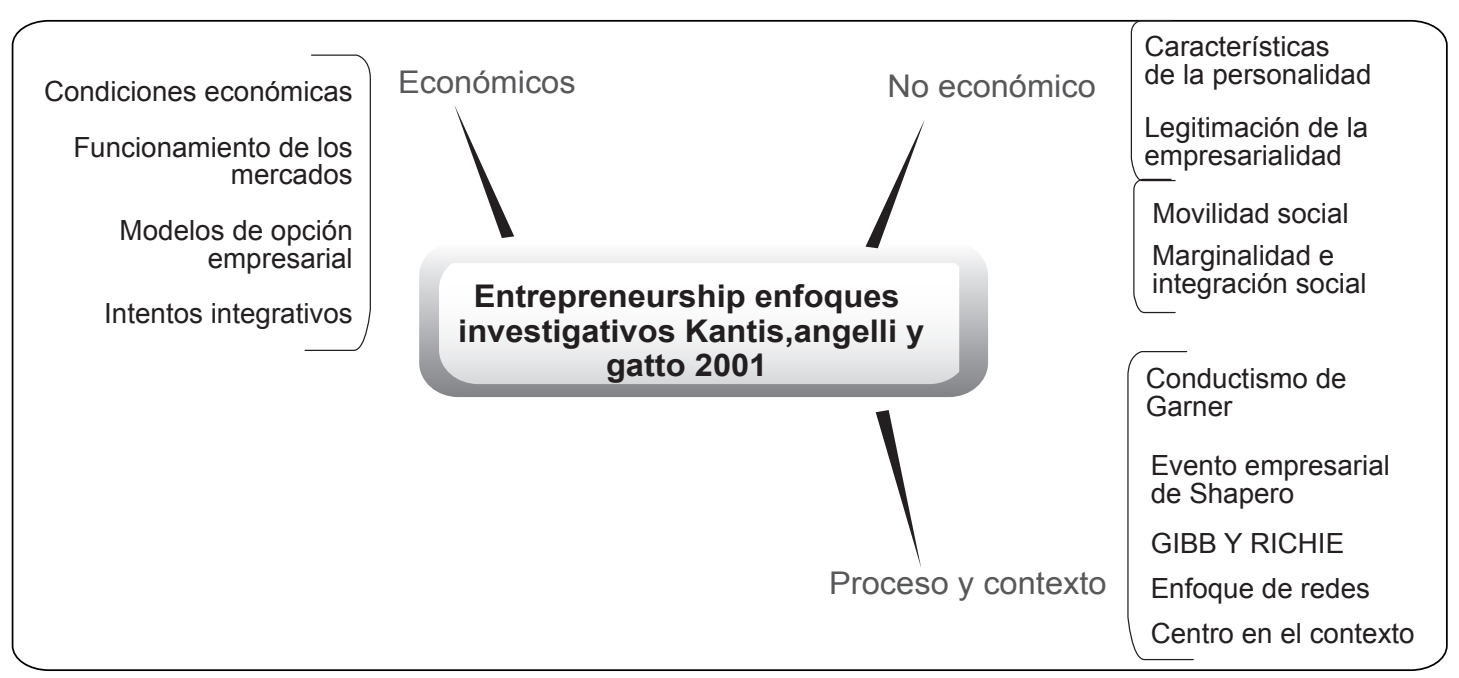

Elaboración propia. Fuente Kantis, Angelli, Gatto, $2001^{3}$

Francisco José González, Tésis doctoral; Universidad de Sevilla 2004. 


\section{Teóricos relevantes del emprendimiento y creación de empresas}

Tomando como base la revisión de la literatura referente a teóricos relevantes del emprendimiento, se realizó esta tabla resumen de los autores más citados en los diferentes journals académicos. Además, se tomo como base a $\mathrm{C}$. Rodríguez \& $\mathrm{M}$. Jiménez. 2005. Profesores de la Universidad Nacional de Colombia. Aquí se presenta un acercamiento importante a la generación de un marco teórico de investigación para la definición y entendimiento del entrepreneurship como campo de estudio.

\begin{tabular}{|c|c|}
\hline R. CANTILLON & $\begin{array}{l}\text { Es un agente que compra medios de producción a ciertos precios, los } \\
\text { transforma y combina en forma ordenada, obtiene de allí un nuevo producto. } \\
\text { El emprendedor a diferencia de otros agentes, toma el riesgo, es decir no } \\
\text { tiene plena certeza, por ende es un agente tomador de riesgos que afecta el } \\
\text { comportamiento de un mercado (citado por Thorton, 1986) }\end{array}$ \\
\hline P. DRUKER & $\begin{array}{l}\text { Plantea al empresario como un innovador. EL empresario innovador busca } \\
\text { el cambio, responde a él y lo explota como una oportunidad (Druker 1985). } \\
\text { Lo define específicamente como el ENTREPRENEUR como el empresario } \\
\text { que es innovador y al entrepreneurship como el empresariado innovador. } \\
\text { Hace diferencia entre un pequeño negocio tradicional y un verdadero } \\
\text { emprendimiento; el cual es aquel que genera cambios en los mercados } \\
\text { (Druker 2002). No todo negocio es un emprendimiento innovador. }\end{array}$ \\
\hline I. KIRZNER & $\begin{array}{l}\text { Plantea que la función empresarial en una sociedad económica no es fácil } \\
\text { de entender. Lo explica mediante la definición del elemento empresarial } \\
\text { en la acción individual humana. Esta se define como el elemento o núcleo } \\
\text { del impulso y perspicacia que lleva a conseguir los recursos y los medios } \\
\text { que se requieren para alcanzar unos objetivos planteados. Para Kitzner el } \\
\text { empresario es quien mueve la sociedad de un estado de quietud (núcleo) } \\
\text { o de no ganancia hacia el equilibrio gradual e incremental a medida que se } \\
\text { perciben precios alternativos lo cual da pie al arbitraje de recursos. }\end{array}$ \\
\hline S.W. KUNKEL & $\begin{array}{l}\text { Q actividad emprendedora es la renovación estratégica. Esta se define como } \\
\text { la gestión del cambio radical y discontinuo. No importa que esta se realice } \\
\text { dentro o fuera de una organización existente y así mismo que la misma } \\
\text { genere una nueva entidad o negocio. }\end{array}$ \\
\hline
\end{tabular}




\begin{tabular}{|c|c|}
\hline A. MARSHAL & $\begin{array}{l}\text { Introduce el concepto de que los factores de producción no son tres sino } \\
\text { cuatro. A los factores tradicionales: Capital, Tierra y Trabajo agrega la } \\
\text { organización y la define como el factor coordinador el cual atrae otros factores } \\
\text { y los agrupa. EL entrepreneurship es el elemento que esta detrás de la } \\
\text { organización manejándola. Plantea que los emprendedores son tienen como } \\
\text { característica básica el liderazgo y actúan en condiciones de incertidumbre. } \\
\text { Aun cuando comparte con otros autores que los emprendedores tienen } \\
\text { ciertas habilidades natas, Plantea que las mismas se pueden aprender y se } \\
\text { pueden adquirir. }\end{array}$ \\
\hline S. MILL & $\begin{array}{l}\text { Ingles de nacimiento lamento que en el idioma ingles no existiera una palabra } \\
\text { con el mismo significado de la palabra francesa ENTREPRENEUR. Enfatizo } \\
\text { la importancia del emprendimiento en el crecimiento económico y que el } \\
\text { desarrollo del entrepreneurship requiere de habilidades no comunes. }\end{array}$ \\
\hline L. MISES & $\begin{array}{l}\text { Para este autor empresario se define como un hombre que actúa de acuerdo } \\
\text { con los cambios que tienen lugar en la información y datos de un mercado. } \\
\text { La actividad empresarial surge de lo que debería hacerse en contraposición } \\
\text { de lo que se está haciendo, de tal forma que se identifican y satisfacen } \\
\text { necesidades de los consumidores. Un factor distintivo del emprendedor es } \\
\text { el estar alerta a los cambios y los percibe como oportunidad. Descubre los } \\
\text { errores e ineficiencias y procura eliminarlos. EL emprendedor descubre lo } \\
\text { que otros han pasado por alto. }\end{array}$ \\
\hline J.B. SAY & $\begin{array}{l}\text { Plantea que un país que cuenta principalmente con comerciantes, industriales } \\
\text { y agricultores será más prospero que uno que cuenta principalmente con } \\
\text { individuos dedicados a las artes o las ciencias. El éxito emprendedor no solo } \\
\text { aporta al individuo sino a la sociedad en general, por esto el emprendedor es } \\
\text { un líder, previsor, tomador de riesgos, evaluador de proyectos que consigue } \\
\text { y moviliza recursos de una zona de bajo rendimiento hacia la productividad. }\end{array}$ \\
\hline J. SHUMPETER & $\begin{array}{l}\text { EL empresario es el destructor creativo. Mediante la innovación entendida } \\
\text { como hacer algo nuevo ya sea un producto, un nuevo proceso o la mejora de } \\
\text { uno ya existente se desequilibran los mercados. El empresario es el motor } \\
\text { de la economía para este autor. }\end{array}$ \\
\hline A. SMITH & $\begin{array}{l}\text { Brevemente trato el tema del entrepreneurship, bajo la palabra inglesa } \\
\text { Business Management, Este autor apunta a obtener el máximo rendimiento } \\
\text { de los recursos y así lograr el equilibrio.. Incluye al empresario en el marco de } \\
\text { los factores externos o fuerzas externas que afectan la dinámica económica. }\end{array}$ \\
\hline H.STEVENSON & $\begin{array}{l}\text { Emprender es perseguir la oportunidad más allá de los recursos disponibles } \\
\text { y controlables en la actualidad. EL empresario es un creador de empresa y } \\
\text { busca la generación de riqueza. Una característica del empresario para que } \\
\text { sea emprendedor es que sea innovador. Para este autor la innovación no } \\
\text { es solo de producto o servicio sino también de procesos o mercados. Hace } \\
\text { diferencia entre el empresario exitoso y el ejecutivo exitoso. }\end{array}$ \\
\hline M. WEBER & $\begin{array}{l}\text { Este autor plantea la tarea del emprendedor como una forma de vida, o una } \\
\text { profesión de vida. Tiene como característica la frugalidad para el logro de } \\
\text { riqueza y por ende tiene una conducta y una ética diferente adquiriendo } \\
\text { códigos específicos para el logro del objetivo. }\end{array}$ \\
\hline
\end{tabular}

Construcción propia basado en C. Rodríguez \& M. Jiménez. 2005. 


\section{Conclusiones}

C omo se puede concluir del análisis de varios autores desde los mismos clásicos de la teoría económica el empresario, o el emprendedor o el manager según sea la definición y traducción, es un elemento o factor importante en la generación de desequilibrios en los mercados y por ende un elemento coadyuvante al desarrollo y crecimiento económico.

Apreciaciones comunes entre los analizados, permiten concluir que la función empresarial en una economía es esencial. Que detrás de una empresa o una organización que genera desequilibrio y genera valor a los consumidores, una innovación; siempre hay un elemento, factor o fuerza y según sea el autor, se define como el entrepreneur o el emprendedor.
Que dichos individuos, entrepreneurs o empresarios, tienen un sistema de creencias, unas habilidades y características particulares que los hace exitosos en el logro de la creación de riqueza. Para algunos de estos autores estas son innatas, pero para la mayoría se pueden enseñar y por ende se pueden aprender, se pueden desarrollar en individuos que no las tienen de manera innata.

A partir de los autores surgen las escuelas de pensamiento y a partir de ellas las teorías, seguido del marco de generación de un marco teórico, para este estudio se describirán y analizarán algunas de las escuelas de pensamiento y las teorías más relevantes para que de ellas se pueda definir el qué enseñar y cómo, a estudiantes de base universitaria para que se tornen empresarios y creen empresas que generen riqueza y por ende desarrollo y crecimiento económico.

\section{co de} Colombia 


\section{Bilbiografía}

Arnal J. (2003): Coordinador. Creación de Empresa: Los mejores Textos. Ariel, España .

Berdugo E., et al (2006): , Formación de Empresarios Emprendimiento y Creación de Empresas. UNIVERSIDAD EAN, Bogota, Colombia.

Bruton G., et.al (2008): "Entrepreneurship in Emerging Economies: Where are today and where should the Research go in the Future". ET\&P Entrepreneurship Theory and Practice, Vol 32. No 1.

Bygrave W.,(1997): The Portable MBA in Entrepreneurship, John Wiley \& Sons, Inc. United States of America.

Cooper. R, Schindler P: Business Research Methods, 7th Edition, Mc Graw Hill, IRWIN.

Cole, A.H., (1949): Entrepreneurship and entrepreneurial history. Change and the entrepreneur, 88-107.

Crissien J., Pérez R (2007): UNIVERSIDAD EAN, 40 Años de Emprendimiento. Universidad EAN, Bogota Colombia.

Crissien J., Guerrero C. (2005):. "Espíritu Emprendedor una verdadera inspiración" Revista Universidad EAN, No 45 Pags 30-54.

Curbelo, J. López J. (2007): Coordinadores. El arte de emprender. Manual para la formacion de emprendedores. Universidad Antonio de Nebrija, Cátedra Emprendedores.

Davidsson P.A. (2005): Researching Entrepreneurship, Springer, P.1. 2005)

Druler P. (1996): La innovación y el empresario innovador, Editorial Norma Cali, Colombia. 
Edmiston K.The Role of Small and Large Business in Economic development. Federal Reserve bank of kansas City, p 74-94, www.kansascityfed,org.

Emory C.(2004):Business Research Methods, 4th edition IRWIN, 1991 Entrepreneurial Leadership, Boston-Londres.

Gartner, W.B. (1988): "Who is an entrepreneur" is the wrong question. American Small Business Journal (spring) 11-31.

Gamboa E. Brouthers E.(2008): "How International is Entrepreneurship?. ET\&P Entrepreneurship Theory and Practice, Vol 32. No. 3 Mayo. Pág 507-528.

Gardner W.B. (1990) What are we talking About when we talk about Entrepreneurship Journal of Business Ventures, p.p 5-28.

Gibb, A. Creating the Entrepreneurial University Worldwide.Do we need a wholly different model of Entrepreneurship?11 This is a shortened and amended version of a policy paper prepared for the UK National Council for Graduate Entrepreneurship (NCGE).

Gardner W.B. (1988): Who is an entrepreneur? S the wrong question, american Journal of Small Business, 12. 11-32.

Gibb A.A. Case Method in entreopreneurship teaching a back ground paper for the EFMD, Unpublished heifeld.

Gibb, A. (1993): Culture and Education, Understanding enterprice education and its links to small businesses. International Business Journal. Vol 11, No 3 April/June

Gibb A. (2004): Creating Conductiva Environment for learning an entrepreneruship living with, delaing with creating and enjoying uncertainty and complexity, 14th annual International Conference, University of Napoli, Federico 11 ( Italy) Napoles.

\section{CAd O88): Creating Conducive Environments for Learning and Entrepreneurship, CIBR A, Stim Llating new business development, M.D.P., ILO, Geneve.}

González, F.J. (2004): Tésis doctoral incidencia del marco institucional en la capacidad emprendedora de los jóvenes empresarios de Andalucía, Universidad de Sevilla. 
Grilo I. and Thurik R. (2004): Determinants of Entrepreneurship In Europe; Max Plank Institute for Research into Economic Systems, Germany.

Gómez L., Martínez J.; Arzuza M. (2006): Política pública y creación de empresas en Colombia. Pensamiento y Gestión No.21. Universidad del Norte Colombia. P 1-25

Hisrich, R. \& Peters M. (1995) Entrepreneurship, (3rd Ed). Burr Ridge, IL Irwin.

Harvard Business School Press Entrepreneurial Venture, , Boston, 1999.

Kantis H. (2005): Desarrollo emprendedor, América Latina y la experiencia internacional, Banco Interamericano de Desarrollo, BID.

Kirzner I.M, (1979), Perception, Oportunity and profit. Studies in the theory of entrepreneurship. The University of Chicago Press, Chicago.

Kalmanovitz S.(2001): Las instituciones y el desarrollo económico en Colombia, Bogotá,Norma.

Kuratko D. (2005): The Emergence Of Entrepreneurship Education; Developement, Trends and Challenges; Entrepreneurship Theory and Practice, September, p577597.

Kuratko, D (2004): Entrepreneurship Educatión in the 21 Century: From legitimitation to leadership, USASBE National Conference.

Kuratko D.F., (1978): Entrepreneurship Education in the 21st Century.

Ladrón de G. (1978): Metodología de la investigación científica, Universidad Santo Tomas, Bogota.

Lumpkin, G.T. \& Dess, G.G. (1996): Clarifying the entrepreneurial orientation construct and liking it to performance. Academy management review, 21 (1), 135-172.

Low, M.B. \& McMillan, I.C. (1988): Entrepreneurship: Past reserarch and future challenges. Journal of Management (14) 139-161. 
Levie J. (1999). Enterpricing Education in Higher Education in England, Departmento of Education and Employment, P40 .

Mason C. (2000). Teaching Entrepreneurship to undergraduates, Lessons from I leading centers of entrepreneurship education, University of Southampton.

McClellamd D. (1961): The achieving society. Harvard University. United States Of America.

McClellamd D., The Achieving Society, MacMillan, New York, 1961.

Méndez C, (1995): Metodología , 2da Edición; Mc graw Hill.

NORTH, D. (1990). Institutions, institutional change and economic performance. Cambridge, University Press.

North, D. (1993). Instituciones, cambio institucional y desempeño económico, México, Fondo de Cultura Económica.

ORTEGON A, CALIXTO N. Experiencias de Formacion de Emprendedores empresrios en Instituciones de Educacion Superior.

PLANT Robert, WILLS Susan, VALLE carlos. "Note to Instructors: Creative Entrepreneurship at iconstruye: A Pan Andean e-procurement Market Maker. ET\&P Entrepreneurship Theory and Practice, Vol 32. No 1 Enero del 2008. Pags 575- 588 RAVIER O. ADRIA, hacia Un estudio comparativo de las Teorias Economicas Defendidas por Joseph Schumpeter y Ludwing Von Mises, Revista Libertas XIII, Instituto Universitario ESEADE, Marzo 2006, www.eseade.edu.ar.

Research, Academy of Management Review, vol. 25 No. 12000.

ROBINSON P., HAYNES MAX, Entrepreneurship Education in America's Major Universties, Journal of Entrepreneurship Theory and Practice, Spring 1991. P 41 ColOmbilarerenusship Text, Cases an Note, Lord Publishing, Dverer.

RONSTADT, R.C. (1985) Training Potential Entrepreneurs. Entrepreneurship: What it is and how to theach it P.Pp 191-204. 
Rodríguez C. \& Jiménez M. (2005) "Emprenderismo, Accion Gubernamental y academia. Revicion de la literatura. INNOVAR Revista de ciencias administrativas y sociales. Escuela de Administracion de Empresas y Contaduria Publica Universidad Nacional de Colombia Julio Diciembre.

SALHMAN, W. et.al (1998) The Entrepreneurial venture, Harvard Busness review.

SCHUMPETER. J.A. (1982) Historia del Análisis Económico, 2da Edición, Ed Arial, Madrid.

SHANE, S; VENKATARAMAN, S.; THe promise of entrepreneurship a field of research, Academy of management Review, vol 25 No 12000.

SHAPERO, A., The entrepreneurial Event in. KENT C.A. (Ed) The environment for entrepreneurship Lexintong Book, D.C. Health 1984.

SHAPERO, Albert, "The entrepreneurial Event in: KENT; C.A. (Ed), The

SOLOMON T. DUFFY S. The state of Entrepreneurship Education in the U.S. A Nationwide Survey and analysis, International Journal of entrepreneurship Education, Pag 65-68, 2002.

STEVENSON, H. H., Babson College Research Conference, Calgary, 1988.

Stevenson, h.h \$ Jarillo j.c. (1990): A paradigm of entrepreneruship: entrepreneursial management- Strategic. Management Journal 11, 10-23.

TARAPUEZ CHAMORRO, E. BOTERO VILLA J.J. Algunos Aportes de los Neoclasicos a la teoría del Emprendedor. Revista de la Facultad de Ciencias Economicas y Administrativas dela Universidad del Quindio, 29-11-2007.

THORTON, M. (1988) The origin of economic theory: A portrait of Richard Cantillon (1680-1734).

TIFFIN Scott. Entrepreneurship In Latin America. Perspectives on Education and Innovation. Praeger Publisehers, United States of America. 2004.

TIMMONS J.; New Venture Creation, Entrepreneruship for the 21st Centrury. IRWIN, Mc Graw Hill. Boston 1999. 
TIMMONS, Jeffry A., New Venture Creation Entrepreneurship for the 21st. Century, VARELA R., BEDOYA O., Modelo Conceptual de desarrollo empresarial basado en Competencias, ICESI; Colombia.

VARELA R., Innovación Empresarial; Arte y Ciencia de la Creación de Nuevas Empresas, Pearson Education, Bogotá Colombia 2001.

VECIANA, Vergés, J.M. (1979) Emprendedor o empresario innovando, Boletín del Centro de Desarrollo del Espíritu Empresarial Universidad ICESI Colombia. No 17 Diciembre.

VECIANA, Vergés, J.M. (1999) "Creación de Empresas como programa de investigación Científica” Revista Europea de Dirección y Economía de la Empresa, Vol 8 N0 3 pp 11-36.

VERHEUL I, W.et.al (2001) Theory of Entrepreneurship: Policies, Institutions and Culture. Timbergen Institute, Discussion Paper; $p$ 1-41.

VESPER K.H. Entrepreneurship Eduaction 1993, University of California 1993.

VESPER K.H. Mc Mullen W.E., Entrepreneurship: today courses, tomorrow.

VESPER K.H., Entrepreneurship Education 1993, University of California.

VESPER K.H., New Venture Strategies, Prentice Hall, Englewood Cliffs, 1990.

Wiklund, j. (1998): Small firm, growth and performance: entrepreneurship and beyond. Doctoral disertation. Jonkoping international business school, jonkoping.

WEST III G. Page, BAMFORD Chales E. MARSDEN Jesse. "Contrasting Entrepreneurial Economic Developement in Emerging Economies: Apllications and Exlensions of Resource Based Theory". ET\&P Entrepreneurship Theory and CActal, 0 32. No 1 Enero del 2008.

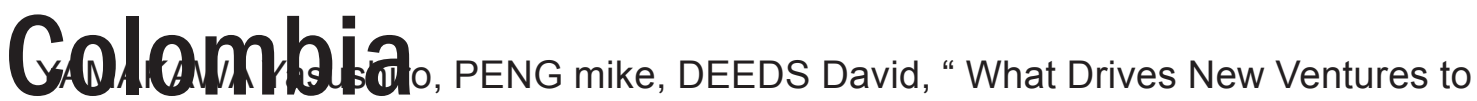
Internacionalize From Emerging to Developed Economies?.”. ET\&PEntrepreneurship Theory and Practice, Vol 32. No 1 Enero del 2008. 